PAPER

\title{
Effects of sleep deprivation on cortical excitability in patients affected by juvenile myoclonic epilepsy: a combined transcranial magnetic stimulation and EEG study
}

\section{P Manganotti, L G Bongiovanni, G Fuggetta, G Zanette, A Fiaschi}

See end of article for authors' affiliations

Correspondence to: Dr Paolo Manganotti, Dipartimento di Scienze Neurologiche e della Visione, Sezione di Neurologia Riabilitativa, Policlinico Borgo Roma, Via delle Menegone, 37134 Verona, Italy; paolo.manganotti@univr.it

Received 8 March 2004 Revised version received 13 June 2005

Accepted 13 June 2005
J Neurol Neurosurg Psychiatry 2006;77:56-60. doi: 10.1136/jnnp.2004.041137

Objective: To investigate the effect of sleep deprivation on corticospinal excitability in patients affected by juvenile myoclonic epilepsy (JME) using different transcranial magnetic stimulation (TMS) parameters. Methods: Ten patients with JME and 10 normal subjects underwent partial sleep deprivation. Motor threshold (MT), motor evoked potential amplitude (MEP), and silent period (SP) were recorded from the thenar eminence (TE) muscles. Short latency intracortical inhibition (SICI) and short latency intracortical facilitation (SICF) were studied using paired magnetic stimulation. TMS was performed before and after sleep deprivation; EEG and TMS were performed simultaneously.

Results: In patients with JME, sleep deprivation induced a significant decrease in $\mathrm{SICl}$ and an increase in SICF, which was associated with increased paroxysmal activity. A significant decrease in the MT was observed. No significant changes in any TMS parameters were noted in normal subjects after sleep deprivation. The F wave was unchanged by sleep deprivation in both control subjects and in patients with JME.

Conclusions: In patients with JME, sleep deprivation produces increases in corticospinal excitability in motor areas as measured by different TMS parameters.
$\mathrm{S}$ leep deprivation is the best method for provoking EEG epileptiform abnormalities ${ }^{1-4}$ and seizures in most types of epilepsy. ${ }^{5}$ Some forms of generalised epilepsy, such as juvenile myoclonic epilepsy (JME), are particularly sensitive to sleep deprivation with motor jerk phenomena. ${ }^{67}$

Nevertheless, the mechanisms underlying the activation of paroxysmal activity, and in particular the myoclonic jerk in JME, are still debated. Some studies have attributed the activation of epileptic patterns to drowsiness and sleep, ${ }^{2}$ while others have concluded that sleep deprivation has a specific activating effect on patients who remain awake during recording. ${ }^{8}$ In animals, sleep deprivation results in a lowering of the thresholds for electroshock convulsions ${ }^{9}$ and kindling ${ }^{10}$ due to a change in the balance between excitatory and inhibitory neurotransmitters. ${ }^{7}$

Transcranial magnetic stimulation (TMS) is a non-invasive method for exploring the excitability of the human motor cortex in vivo. Several TMS elements contribute to stimulation of the motor threshold (MT), during which the silent period (SP) may be assessed by the single pulse technique. The paired pulse method allows measurement of the so called short latency intracortical inhibition (SICI) and short latency intracortical facilitation (SICF) at short interstimulus intervals (ISIs) (1-25 ms). ${ }^{11}$ Intracortical inhibition and facilitation are thought to reflect the excitability of separate populations of interneurones that are intrinsic to the cortical motor area. In fact, a reduction in cortical inhibition has been observed in different forms of epilepsy including progressive myoclonic epilepsy, ${ }^{12-14} \mathrm{JME},{ }^{15-17}$ generalised epilepsy, ${ }^{18}$ and partial epilepsy. ${ }^{19}$ All these studies have been performed in epileptic patients when awake after normal sleep.

The important effect of sleep and sleep deprivation on cortical excitability investigated by TMS has been recently investigated in different studies with contrasting findings. In normal subjects, sleep deprivation produces a mild decrease in cortical excitability during night time that is probably related to drowsiness, although no differences in TMS values have been observed either before or after sleep deprivation. ${ }^{20}$ It has been reported that corticospinal fibres are normally activated by magnetic stimulation, while motor excitability is decreased during the different sleep stages. ${ }^{21}{ }^{22}$ In contrast, other authors ${ }^{23}$ have observed that in normal subjects, $24 \mathrm{~h}$ of sleep deprivation produces an increase in motor excitability as studied by TMS in two sessions, namely before and after sleep deprivation.

The different effects on motor excitability of sleep deprivation could be due to the different methods and objectives of the studies. Nevertheless, the sensitivity of TMS to sleep deprivation has introduced a new feature in clinical research as it allows one to evaluate hypotheses regarding changes in motor excitability in epileptic patients after activation tests that are commonly used in clinical practice. To date, no information exists on the possible changes in motor cortex excitability in epileptic patients after sleep deprivation. The main goal of the present study was to investigate the excitability of the motor cortex after sleep deprivation in patients affected by JME who are particularly sensitive to this activation test.

\section{METHODS}

\section{Subjects}

Ten patients (eight women, two men; age range 16-33 years) affected by JME were compared to 10 healthy age matched control subjects (five women, five men; age range 1830 years). The control subjects had no history of seizures. Patients with JME had a history of seizures ranging from 2 to 7 years, and the age at onset of seizures ranged from 14 to

Abbreviations: CMAP, compound motor action potential; ISI, interstimulus interval; JME, juvenile myoclonic epilepsy; MEP, motor evoked potential; MT, motor threshold; SICF, short latency intracortical facilitation; SICl, short latency intracortical inhibition; SP, silent period; $T E$, thenar eminence; TMS, transcranial magnetic stimulation; VAS, visual analogue scale 
18 years. All these patients reported the presence of myoclonic jerks characterised by short, sudden, arrhythmic, and symmetric muscle movements mainly affecting the shoulders and arms. Myoclonic jerks occurred frequently and briefly after awakening. Patients always consciously experienced these jerks and showed mild symptoms of absence as reported by relatives. Rare, generalised tonicclonic seizures were also described, particularly after sleep deprivation. The EEG recording at rest was characterised by occasional bursts of polyspikes and waves on a normal rhythm background. Eight patients with JME were being treated with antiepileptic drugs (phenobarbital and valproate), while two patients with JME were not receiving pharmacological treatment during the study. The drugs were taken at the same time on each day that measurements were recorded, although we did not measure plasma levels. Neuroradiological studies (MRI) were normal in all patients. The study was approved by the local ethics committee. All individuals provided written informed consent.

\section{Experimental design}

All patients were admitted to the hospital. Sleep deprivation was experienced by both patients and controls who were all asked to stay awake from midnight until morning; napping was forbidden. Sleep deprivation was monitored by nurses. The first TMS recording was carried out between 9 and 10 a.m. on the morning before sleep deprivation and the second TMS recording was carried out at the same time on the morning after partial sleep deprivation. In all patients, the EEG recording was carried out before, during, and after each TMS recording using the coregistration EEG-TMS method..$^{22}$

Sleep deprivation in control subjects was monitored in the laboratory. Subjective sleepiness after sleep deprivation in controls and patients was evaluated in the morning before the TMS recording using a $10 \mathrm{~cm}$ visual analogue scale (VAS). ${ }^{24}$

\section{Magnetic stimulation}

All individuals were seated in an armchair with the elbow semi-flexed; the forearm was pronated, fully relaxed, and supported by the arm of the chair. Control and conditioned motor evoked potentials (MEPs) were recorded from the right thenar eminence (TE) muscles by surface electrodes in all subjects. All individuals were asked to keep their eyes open. The amplified and band pass filtered ( $50 \mathrm{~Hz}$ to $5 \mathrm{KHz}$ ) EMG signal was fed into a Medelec Machine (Oxford, UK) with a sampling rate of $10000 \mathrm{~Hz}$. An auditory feedback EMG signal was produced to ensure complete voluntary relaxation of the target muscles. Trials in which voluntary EMG activity or fasciculation occurred were discarded from further analysis.

A circular magnetic coil was applied using a Magstim 200 magnetic stimulator (Magstim, Whitland, Dyfed, UK). Intracortical excitability was studied by paired TMS. For this purpose, two magnetic stimulators were connected to one circular coil ( $9 \mathrm{~cm}$ diameter) by mean of a Bistim device (Magstim).

The optimal scalp position for the magnetic coil, defined as the site where moderate suprathreshold stimulation consistently yielded the largest MEP in TE muscles, was determined by moving the coil around the vertex in order to find the presumed hand area of the motor cortex contralateral to the recorded TE muscles. A constant position of the coil relative to the skull throughout the session was ensured by using a scalp cap, where the site of stimulation was marked.

The different TMS parameters used to investigate motor system excitability are described below.

(i) The MT intensity was defined as the lowest stimulator output intensity capable of inducing MEPs of at least a $50 \mu \mathrm{V}$ peak-to-peak amplitude in the relaxed TE muscles in at least five of 10 trials. ${ }^{25} 26$

(ii) Peak-to-peak MEP amplitudes were measured in the resting TE muscles at stimulus intensities of $110 \%, 120 \%$, and $130 \%$ of the resting MT. A total of seven stimuli were delivered to each muscle in each session. The size of the MEPs was measured as a percentage of the compound motor action potential (cMAP) elicited by peripheral stimulation of the median nerve. ${ }^{26}$

(iii) The duration of the SP was determined at stimulus intensities of $110 \%, 120 \%$, and $130 \%$ of the MT in TE muscles performing a mean voluntary contraction of about $10 \%$ of maximum voluntary contraction. Voluntary contraction was measured using a dynamometer constructed in our laboratory. The SP was measured in the single trial rectified EMG recordings from the end of the preceding MEP to the onset of sustained voluntary EMG activity. The averages of seven trials were calculated for each stimulus intensity. ${ }^{27}$

(iv) Paired TMS was performed using standardised paradigms in order to investigate SICI and SICF. ${ }^{11} \mathrm{~A}$ subthreshold conditioning stimulus was set at $70 \%$ of MT and was delivered through the same magnetic coil at ISIs of $1,2,3,4,10$, and $15 \mathrm{~ms}$ before a suprathreshold test stimulus. The test stimulus intensity was adjusted to $120 \%$ above the MT. ${ }^{11}$ This procedure allows the measurement of SICI (ISI ranging from 1 to $4 \mathrm{~ms}$ ) and SICF (ISI ranging from 10 to $15 \mathrm{~ms}$ ). The averages of five trials were calculated for each ISI, and averages from 1 to $4 \mathrm{~ms}$ and from 10 to $15 \mathrm{~ms}$ were obtained in order to produce a single measure for SICI and SICF, respectively.

Finally, in order to evaluate spinal excitability, we studied the F wave amplitude. F responses were recorded from the TE and elicited by supramaximal stimulation of the median nerve at the wrist once every $1 \mathrm{~s}$. For each set of seven stimuli, we measured the mean $M$ wave peak-to-peak amplitude and the mean F wave peak-to-peak amplitude. Seven $\mathrm{F}$ responses were collected for each recording session.

\section{EEG recording}

In all individuals, the EEG recording was carried out before and after sleep deprivation as well as before, during, and after the TMS recording in order to monitor the effects of TMS stimulation on EEG activity. The EEG-TMS coregistration lasted $30 \mathrm{~min}$, while each EEG recording before and after the TMS lasted $15 \mathrm{~min}$. EEG recording was carried out in conscious individuals with their eyes open in order to avoid subjective drowsiness as much as possible. The EEG recording was carried out without any additional provocation (for example, intermittent photic stimulation or hyperventilation).

EEG was recorded using a 19 channel digital electroencephalograph (Micromed Instruments, Mogliano Veneto, Italy). The electrode array was in accordance with the International 10-20 system of electrode placement and was mounted on an elastic cap resistant to magnetic pulses (Micromed Instruments). A monopolar montage with a biauricular reference was used. Electrode impedance was below $5 \mathrm{~K} \Omega$. EEG signals were recorded with a band pass of 1.6-120 Hz and a sampling rate of $1024 \mathrm{~Hz}$. Coregistration was performed by delivering the TMS using the EEG cap. The artefact of magnetic stimulation on the EEG activity lasted from 20 to $40 \mathrm{~ms}^{22}$

Paroxysmal activity was quantified on the basis of the number of spikes, spikes and waves, and bursts of high amplitude sharp waves on $30 \mathrm{~min}$ of EEG recordings performed before and after sleep deprivation. The presence and the frequency of the paroxysmal activity were considered for each recording. 


\section{Statistical analysis}

The SPSS program (release 11.0.0; SPSS, Chicago, IL) was used for statistical analysis To compare the performance of epileptic patients and control subjects for each parameter, statistical analyses were performed using GreenhouseGeisser corrected repeated measures ANOVAs. Each ANOVA had one between-subjects factor, "group" (that is, patient or control), and a within-subject factor "time" (that is, pre and post sleep deprivation). To compare within each group and between groups, significant differences before and after the sleep deprivation, and all significant two way interactions (that is, group $\times$ time), post hoc analyses with pairwise paired $t$ tests were performed.

\section{RESULTS \\ Effects of sleep deprivation comparing epileptic patients and controls}

There were no significant differences in most TMS parameters (SP, MEP amplitude) or in F wave amplitude and latency between controls and epileptic patients (fig l, table 1). SICI and SICF parameters were grouped together for ANOVA analysis. With regard to the SICI, repeated ANOVA measures showed the following statistically significant main effects and interactions: the "time" main effect, with $F_{1,18}=5.276$, $\mathrm{p}<0.01$, and the group $\times$ time interaction, with $F_{1,18}=11.327$, $\mathrm{p}<0.005$. Post hoc $t$ tests for the two way interaction showed that the baseline SICI values were significantly reduced in all patients with JME compared to controls $(p<0.001)$. Moreover, there was a statistically significant sleep deprivation effect in all patients with JME $(\mathrm{p}<0.001)$ but not in control subjects.

The SICF parameter showed the following statistically significant interactions: group $\times$ time, with $F_{1,18}=9.174$, $\mathrm{p}<0.0$ l. Post hoc analyses showed that no significant differences were present in SICF between controls and epileptic patients. However, there was a sleep deprivation effect, with statistically significant augmentation of the SICF in the group of patients with JME $(\mathrm{p}<0.005)$ but not in controls.

The following statistically significant effects and interactions were found in the MT parameter: the "time" main effect, with $F_{1,18}=63.158, \mathrm{p}<0.0001$, and the group $\times$ time interaction, with $F_{1,18}=90.947, \mathrm{p}<0.0001$. Post hoc analyses showed no significant differences between the two groups at baseline with respect to MT values. However, there was a statistically significant sleep deprivation effect in all patients with JME with a reduction in MT $(p<0.001)$ which was not observed in control subjects.

Eight of 10 patients with JME were being treated with anticonvulsant drugs (phenobarbital and valproate); the

\begin{tabular}{|c|c|c|c|c|c|c|c|c|}
\hline & \multicolumn{4}{|l|}{ JME } & \multicolumn{4}{|c|}{ Controls } \\
\hline & \multicolumn{2}{|l|}{ Before } & \multicolumn{2}{|l|}{ After } & \multicolumn{2}{|l|}{ Before } & \multicolumn{2}{|l|}{ After } \\
\hline & Mean & SD & Mean & SD & Mean & SD & Mean & SD \\
\hline MT (\%) & $45^{\star}$ & 5 & $40^{*}$ & 3 & 45 & 3 & 46 & 4 \\
\hline MEP amp (\%) & 10 & 4 & 11 & 3 & 9 & 5 & 8 & 3 \\
\hline SP $10 \%$ (ms) & 40 & 8 & 42 & 7 & 41 & 6.5 & 43 & 7 \\
\hline SP $20 \%$ (ms) & 80 & 10 & 75 & 9 & 79 & 8 & 80 & 9 \\
\hline SP $30 \%$ (ms) & 155 & 13 & 140 & 9 & 152 & 8 & 155 & 10 \\
\hline $\mathrm{SICl}(\%)$ & $44^{*}$ & 8 & $62^{\star}$ & 14 & 28 & 6 & 24 & 3 \\
\hline SICF (\%) & $107^{*}$ & 18 & $120^{*}$ & 24 & 120 & 12 & 119 & 9 \\
\hline $\mathrm{F}$ wave $(\mu \mathrm{V})$ & 200 & 50 & 250 & 100 & 200 & 100 & 270 & 90 \\
\hline
\end{tabular}

remaining two patients were not under pharmacological treatment. The decrease in SICI observed after sleep deprivation was larger in untreated patients compared to pharmacologically treated patients (fig 1). Sleep deprivation did not produce any significant changes in SICI, SICF, or MT in comparison with baseline values in normal subjects (fig 1 , table 1).

All individuals complained of tiredness and drowsiness at the time of the test recording after sleep deprivation. The average VAS score ranged from 2 to 3, meaning that somnolent subjects were still sufficiently alert and were capable of following the experiment instructions. No subjects had seizures after sleep deprivation. No paroxysmal activity was observed in normal subjects in the EEG recording after sleep deprivation and no significant changes were noted in the $\mathrm{F}$ wave amplitude.

In seven patients, the EEG recording performed before TMS showed an increase in paroxysmal activity compared to the EEG recording at baseline. The paroxysmal activity was characterised by repetitive or single bursts of polyspikes and waves. After 30 min of EEG recording, these patients showed an increase from a mean of $4 \pm 1$ to $13 \pm 3$ polyspikes bursts.

No patients complained of seizures during the TMS recording either before or after sleep deprivation and no seizures were reported in the days following the TMS recordings. Likewise, none of the patients complained of discomfort during the TMS recording either before or after sleep deprivation and no myoclonic jerks were observed in patients with JME during TMS recording. As reported in our previous study, ${ }^{16}$ most of the patients with JME reported that the proprioceptive sensations of twitching elicited normally by TMS stimulation were similar as subjective sensations to those occurring spontaneously after awakening. However, no myoclonic jerks were observed during or after TMS. No paroxysmal activity was produced or observed during or immediately after TMS during EEG coregistration. The average VAS score ranged from 2 to 3 . At the time of the test recording after sleep deprivation, all individuals complained of tiredness and drowsiness but were still sufficiently alert to follow the experiment instructions. No significant differences were noted in a subjective evaluation of drowsiness by VAS between patients and control subjects.

\section{DISCUSSION}

The main finding of the present study is that sleep deprivation induced changes in patients with JME in most of the TMS variables representing different aspects of the primary motor cortex excitability. Loss of SICI, increases in SICF, and decreases in the MT were noted after sleep deprivation over the motor areas, while the indexes of spinal excitability ( $\mathrm{F}$ waves) and neuromuscular excitability ( $\mathrm{M}$ waves) remained unaltered.

Importantly, no changes were noted in TMS parameters in normal subjects before or after sleep deprivation in the morning, in agreement with previous studies..$^{20}$ The transitory decrease in motor excitability observed during the night of sleep deprivation in the previous study ${ }^{20}$ was probably related to the drowsiness of the subjects, and was not addressed in the present series of experiments. In fact, a decrease in motor excitability during drowsiness and sleep has been recently documented in studies coregistering EEG and TMS in normal subjects. ${ }^{21} 22$

The coregistration of EEG and TMS also allowed us to observe an increase in motor excitability in those few patients with no significant increases in EEG paroxysmal activity after sleep deprivation.

Mild, significant changes in MTs were noted after sleep deprivation in JME patients but not in controls. The MT most likely reflects the excitability of the neuronal membranes. ${ }^{26}$ 

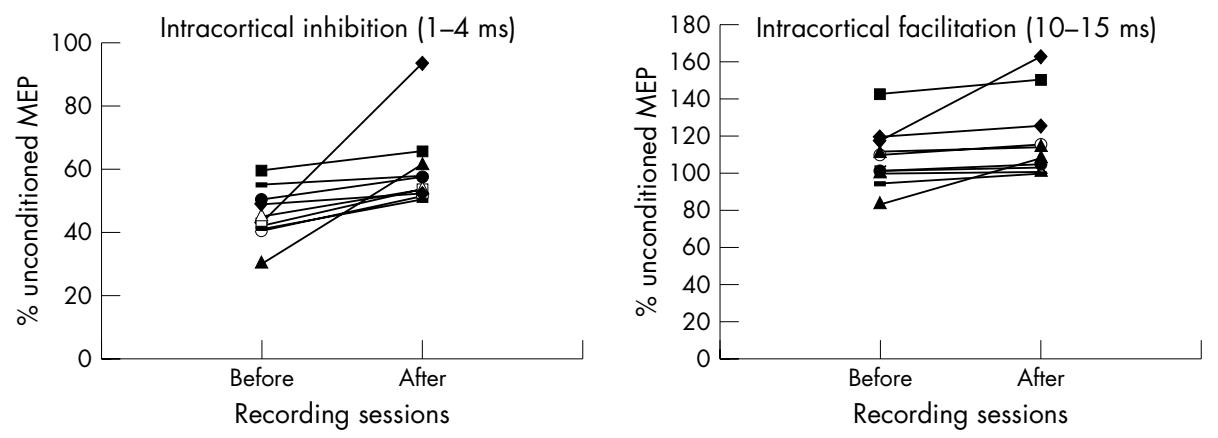

Epileptic patients
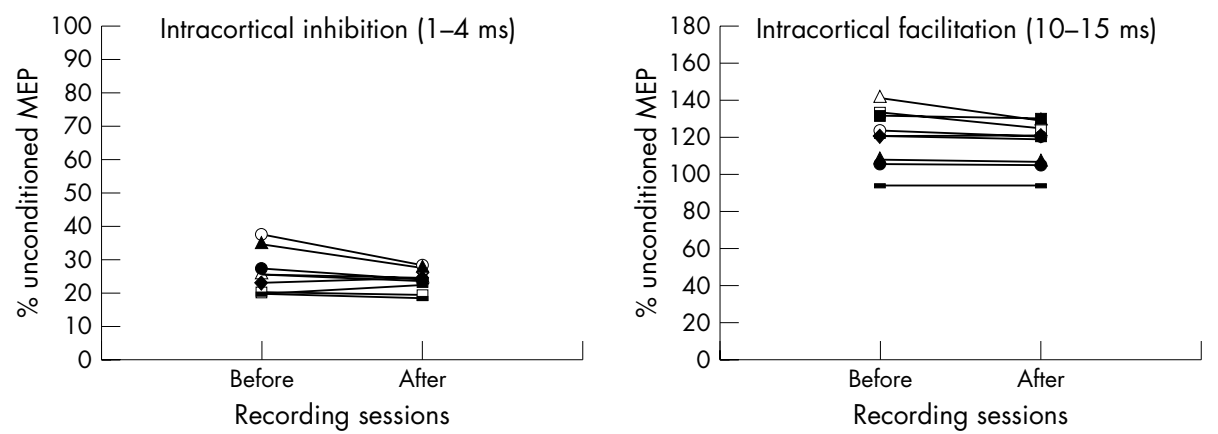

Healthy controls

Figure 1 Individual SICl and SICF recorded from the right TE muscles in 10 epileptic patients (above) and in 10 healthy controls (below) before and after sleep deprivation. The SICl is averaged across inhibitory ISIs of 1-4 ms, while the SICF is averaged across ISIs of 10-15 ms. Both are expressed as the ratio of conditioned MEP to unconditioned MEP amplitudes (y axis). The different symbols indicate the various subjects and are the same in all figures. Intracortical inhibition was reduced in 10 patients affected by myoclonic epilepsy. In healthy controls no consistent changes across the individual data were observed in either the SICl or SICF after sleep deprivation.

We assume that sleep deprivation in patients affected by JME, but in not normal subjects, had an effect on the membrane of the nerve cells that are presynaptic to the corticospinal neurone and usually excited by TMS. ${ }^{25}$

Sleep deprivation did not alter the SP in either normal subjects or epileptic patients. ${ }^{27}$ The SP could be the result of simultaneous excitation and inhibition and may therefore not be the most appropriate parameter for studying motor cortex inhibition.

Sleep deprivation produced loss of intracortical inhibition and mild increase in facilitation in epileptic patients but not in normal subjects.

The first ( $1-5 \mathrm{~ms}$ ) phase, SICI, is thought to depend upon GABAergic mechanisms. ${ }^{26}{ }^{28} 29$ The SICF is thought to result from the activation of excitatory interneurones with glutamate mechanisms. ${ }^{126} 30$ The reduction in early SICI with mild changes in SICF at rest in the present series of epileptic patients provides further evidence in support of the hypothesis that inhibitory mechanisms may be altered in epilepsy. ${ }^{631-33}$

The increase in motor excitability after sleep deprivation could be related to changes in GABAergic mechanisms and cholinergic mechanisms, closely related to hypnogenic mechanisms. ${ }^{34}$

Antiepileptic drugs can modify the TMS parameters, ${ }^{26}{ }^{35-37}$ although in JME patients the presence of antiepileptic drugs only partially changed abnormal motor inhibition. ${ }^{16}$ No significant changes were noted in the $F$ wave in either control subjects or epileptic patients, thus suggesting an action of sleep deprivation above the spinal level.

Nevertheless, we cannot rule out the involvement of subcortical structures as in basal ganglia pathology (for example, Parkinson's disease).$^{38}$ Evidence has been found for a relationship between spindle production and epileptic activity $^{39} 40$ as a cortical response to intrinsic thalamocortical volleys. ${ }^{41}$ Finally, no seizures were reported in patients after sleep deprivation during or after single and paired pulse TMS. ${ }^{42} 43$

In conclusion, sleep deprivation is a significant activator that produces an increase in motor excitability in epileptic patients, most likely related to a decrease in GABAergic mechanisms. The use of paired TMS as a research tool in sleep disorders merits further investigation.

\section{Authors' affiliations}

P Manganotti, A Fiaschi, G Fuggetta, Dipartimento di Scienze Neurologiche e della Visione, Servizio di Neurologia ad Indirizzo Riabilitativo, University of Verona, Verona, Italy

L G Bongiovanni, G Zanette, Dipartimento di Scienze Neurologiche e della Visione, Sezione di Neurologia, University of Verona, Verona, Italy

Competing interests: none declared

\section{REFERENCES}

1 Bennett DR. Sleep deprivation and major motor convulsions. Neurology 1963;13:953-8.

2 Pratt KL, Mattson RH, Weikers NJ, et al. EEG activation of epileptics following sleep deprivation: a prospective study of 114 cases. Electroencephalogr Clin Neurophysiol 1968;24:11-15.

3 Jovanovic UJ. General consideration of sleep and sleep deprivation. Epilepsy Res Suppl 1991;2:205-15.

4 King MA, Newton MR, Jackson GD, et al. Epileptology of the first seizure presentation: a clinical electroencephalographic and magnetic resonance imaging study of 300 consecutive patients. Lancet 1998;352:1007-1 1.

5 Dinner DS. Effect of sleep on epilepsy. J Clin Neurophysiol 2002; 19(6):504-13

6 Janz D. Epilepsy with grand mal on awakening and sleep-waking cycle. Clin Neurophysiol 2000;111(suppl 2):S103-10.

7 Rodin E. Sleep deprivation and epileptological implications. In: Degen R, Rodin EA, eds. Epilepsy, sleep and sleep deprivation. Epilepsy Res $1991 ; 2: 265-73$. 
8 Naitoh P, Dement W. Sleep deprivation in humans. In: Remoond A, ed. Handbook of electroencephalography and clinical neurophysiology. Amsterdam: Elsevier, 1974:146-51.

9 Cohen HB, Dement WC. Sleep: changes in threshold to electroconvulsive shock in rats after deprivation of "paradoxical" phase. Science 1965;150:1318-19.

10 Shouse MN. Sleep deprivation increases thalamocortical excitability in the somatomotor pathways, especially during seizure-prone sleep or awakening states in the feline seizure models. Exp Neurol 1988;99:664-7.

11 Kujirai T, Caramia MD, Rothwell JC, et al. Cortico-cortical inhibition in human motor cortex. J Physiol (Lond) 1993;471:501-19.

12 Brown P, Ridding MC, Werhahn KJ, et al. Abnormalities of balance between inhibition and excitation in the motor cortex of patients with cortical myoclonus. Brain 1996;119:309-17.

13 Reutens DC, Puce A, Burkovic SF. Cortical hyperexcitability in progressive myoclonus epilepsy: a study with transcranial magnetic stimulation. Neurology 1993;43:186-92.

14 Manganotti P, Tamburin S, Zanette G, et al. Hyperexcitable cortical response in progressive myoclonic epilepsy: a TMS study. Neurology 2001;57:1793-9.

15 Caramia MD, Ciccinelli P, Paradiso C, et al. "Excitability" changes of muscular responses to magnetic brain stimulation in patients with central motor disorders. Electroencephalogr Clin Neurophysiol 1991;81:243-50.

16 Manganotti P, Bongiovanni LG, Zanette G, et al. Early and late intracortical inhibition in juvenile myoclonic epilepsy. Epilepsia 2000;41(9):1129-38.

17 Manganotti P, Tamburin S, Bongiovanni LG, et al. Motor responses to afferent stimulation in juvenile myoclonic epilepsy. Epilepsia 2004;45(1):77-80.

18 Brodtmann A, Macdonell RA, Gilligan AK, et al. Cortical excitability and recovery curve analysis in generalized epilepsy. Neurology 1999.53(6):1347-9.

19 Cantello R, Civardi C, Cavalli A, et al. Cortical excitability in cryptogenic localization related epilepsy: interictal transcranial magnetic stimulation. Epilepsia 2000;41:694-704

20 Manganotti P, Palermo A, Patuzzo S, et al. Decrease in cortical excitability in human subjects after sleep deprivation. Neurosci Lett 2001;304:153-6.

21 Grosse P, Khatami R, Slih A, et al. Cortical excitability in human sleep as assessed by transcranial magnetic stimulation. Neurology 2002;59(12):326-42

22 Manganotti $P$, Fuggetta $G$, Fiaschi $A$. Changes of motor cortical excitability in human subjects from wakefulness to early stages of sleep: a combined transcranial magnetic stimulation and electroencephalographic study. Neurosci Lett 2004;362(1):31-4

23 Civardi C, Boccagni C, Vicentini R, et al. Cortical excitability and sleep deprivation: a transcranial magnetic stimulation study. Neurol Neurosurg Neuropsychiatry 2001;71(6):809-12.

24 Dumont M, Macchi MM, Carrier J, et al. Time course of narrow frequency bands in the waking EEG during sleep deprivation. Neuroreport 1999:10:403-7.

25 Rossini PM, Barker AT, Berardelli A, et al. Non invasive electrical and magnetic stimulation of the brain, spinal cord and roots: basic principles and procedures for routine clinical application. Report of an IFCN Committee. Electroencephalogr Clin Neurophysiol 1994;91:79-92.

26 Ziemann U, Lonnecker S, Steinhoff BJ, et al. Effects of antiepileptic drugs on motor cortex excitability in humans: a transcranial magnetic stimulation study. Ann Neurol 1996:40:367-78.

27 Hallett M. Transcranial magnetic stimulation. Negative effects. Adv Neurol 1995;67:107-13.

28 Ziemann U, Lonnecker S, Steinhoff BJ, et al. Inhibition of human motor cortex by ethanol: a transcranial magnetic stimulation study. Brain 1995; 118:1437-46.

29 Siebner HR, Dressnandt J, Auer C, et al. Continuous intrathecal baclofen infusions induced a marked increase of transcranially evoked silent period in a patient with generalized dystonia. Muscle Nerve 1998;21:1209-12.

30 Liepert J, Schwenkreis $P$, Tangenthoff $M$, et al. Glutamate antagonist riluzole suppresses intracortical facilitation. J Neural Transm 1997;104:1207-14.

31 Krnjevic K. Significance of GABA in brain function. In: Tunnicliff G, Raess BU, eds. GABA mechanisms in epilepsy. New York: Wiley-Liss, 1991:47-87.

32 Olsen RW, Avoli M. GABA and epileptogenesis. Epilepsia 1997:38:399-407.

33 Janz D. The idiopathic generalized epilepsies of adolescence with childhood and juvenile age of onset. Epilepsia 1997;38:4-11

34 Velazquez-Moctezuma J, Shiromani PJ, et al. Acetylcholine and acetylcholine receptor subtypes in REM sleep generation. In: Aquilonius SM, Gilberg PG, eds. Progress in brain research. Vol 84. Amsterdam: Elsevier, 1990:407-13.

35 Hufnagel A, Elger CE, Marx W, et al. Magnetic motor evoked potentials in epilepsy: effects of the disease and of anticonvulsant medication. Ann Neurol 1990;28(5):680-6.

36 Reutens DC, Berkovic SF, Macdonell RA, et al. Magnetic stimulation of the brain in generalized epilepsy: reversal of cortical hyperexcitability by anticonvulsants. Ann Neurol 1993;34:351-5.

37 Manganotti P, Bongiovanni LG, Zanette GP, et al. Cortical excitability in patients after loading doses of lamotrigine: a study with magnetic brain stimulation. Epilepsia 1999:40(3):316-21.

38 Ridding MC, Inzelberg R, Rothwell JC. Changes in excitability of motor cortical circuitry in patients with Parkinson's disease. Ann Neurol 1995;37:181-8.

39 Gloor P. Generalized epilepsy with spike and wave discharge: a reinterpretation of its electrographic and clinical manifestations. Epilepsia 1979:20:571-88.

40 Kostopolous G, Gloor P, Pellegrini A, et al. A study of the transition from spindles to spike and wave discharges in feline generalized penicillin epilepsy: microphysiological features. Exp Neurol 1981;73:55-73.

41 Steriade M, Contreras D. Relationship between cortical thalamic cellular events during transition from sleep patterns to paroxysmal activity. J Neurosci 1995; 15:623-42.

42 Schrader LM, Stern JM, Koki L, et al. Seizure incidence during single and paired pulse transcranial magnetic stimulation (TMS) in individuals with epilepsy. Clin Neurophysiol 2004;115:2728-37.

43 Tassinari CA, Cincotta M, Zaccara G, et al. Transcranial magnetic stimulation and epilepsy. Clin Neurophysiol 2003;1 14:777-98. 